\title{
Fast and Effective Retrieval of Plant Leaf Shapes
}

\author{
Bin Wang ${ }^{1,2}$ and Yongsheng Gao ${ }^{2}$ \\ ${ }^{1}$ Key Laboratory of Electronic Business, Nanjing University of Finance and \\ Economics, Nanjing 210096, China \\ wangbin@njue.edu.cn \\ ${ }^{2}$ School of Engineering, Griffith University, QLD 4111, Australia \\ \{bin.wang,yongsheng.gao\}@griffith.edu.au
}

\begin{abstract}
In this paper, a novel shape description and matching method based on multi-level curve segment measures (MLCSM) is proposed for plant leaf image retrieval. MLCSM extracts the statistical features of shape contour via measuring the curve bending, convexity and concavity of the curve segments with different length of shape contour to describe the shape. This method not only finely captures the global and local features, but also is very compact and has very low computational complexity. The performance of the proposed method is evaluated on the widely used Swedish leaf database and the leaf databases collected by ourselves which contains 1200 images and 100 plant leaf species. All the experiments show the superiority of our method over the state-of-the-art shape retrieval methods.
\end{abstract}

\section{Introduction}

From the theory of plant shape taxonomy, the shape of plant leaf provides valuable cues for plant identification. Unlike plant flower which has complex 3D structure and can only be obtained at blooming season, plant leaf is thin and flat, and can been found everywhere and every time, therefore, it has been widely used in computer-aided plant species identification. Retrieving similar leaf shapes from the leaf image database is a very important and challenge task. Its difficulties lies in: (1) there are about 400,000 species of plants over the world $[1]$ and a large amount of leaf images (millions of samples) will be stored in the databases which makes retrieve speed (i.e. retrieve efficiency ) suffer from challenge; (2) Many plants's leaf shapes have tiny difference from each other which are very difficult to discriminate them. Fig. 1 lists five plant leaves with very similar shapes which belong to different plant species.

In the past three decades, many shape description and matching methods have been proposed for image retrieval. According to whether shape features are extracted from the contour only or from the whole region, Zhang et al. [2] classified them into region-based methods and contour-based methods. Since the contour of the leaf shape contains the all shape information, we focus on the contour-based methods. Fourier descriptors (FDs) [3] is a classical shape description method, in which a shape signature, such as curvature, tangent angles, 


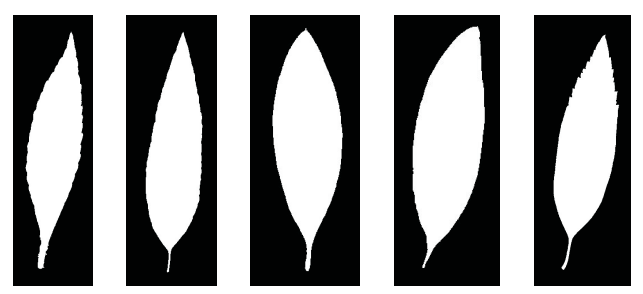

Fig. 1. Five leave images from five plant species which have very similar shapes.

complex coordinates, or centroid distance, is firstly extracted from the shape contour, and a discrete Fourier transform (DFT) is then applied to the signature to generate Fourier coefficients as the shape descriptors. The length of the feature vector for FDs is usually no more than 20 and the measure of shape similarity is a metric distance between the feature vectors. These desirable properties make FDs achieve a high level of matching efficiency. To further study the performance of FDs for the shape retrieval and classification, some works [4-6] have compared FDs with the other shape description methods including autoregressive modeling, CSS (curvature scale space), and moment descriptors and show that among all the competing methods, FDs is the best one for shape retrieval and classification. In [7], Zhang et al. conducted a large amount of shape retrieval experiments to evaluate the performance of different FDs derived from various shape signatures and report that the centroid distance signature is the best one to be used to generate FDs with higher retrieval performance. Although the above methods have achieved promising retrieval performance, they are not suitable for leaf image retrieval because of their poor ability of capturing local shape features. We use an example to address this problem.

In Fig. 2. we list three leaf image (a,b,c), where a and b belong to the same class and c belong to another class, in the right figure, we plot the curves of their centroid distance signatures. We carefully compare these signatures and find that the signature of leaf $\mathrm{A}$ close to leaf $\mathrm{C}$ rather than close to leaf $\mathrm{B}$. Therefore FDs method matches leaf a to leaf $\mathrm{c}$ rather than to leaf $\mathrm{b}$ which gives a false matching,

In recent years, to improve the retrieval accuracy, many methods dedicate to find a one-to-one dense point correspondence between two matched shape contours and the obtained optimal correspondence is used to define the dissimilarity between two shapes. Among these methods, Shape context (SC) [8] is a classical method and continues attracting many researchers' attentions. SC builds a histogram for each contour point to describe the relative distribution of the remaining points to that point, and these histograms are used to find the correspondence in a point-by-point manner. To make shape descriptors articulation invariant, Lin et al. [9] replaced Euclidean distance with inner-distance (ID), which is defined as the shortest path between two contour points within the shape boundary, to extend the SC method as a novel shape descriptor called IDSC. Adamek et al. [10] proposed a muti-scale shape representation method, 


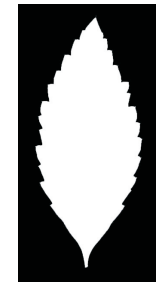

a

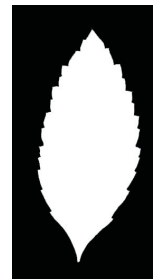

b

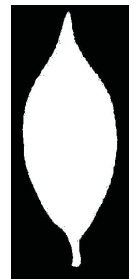

C

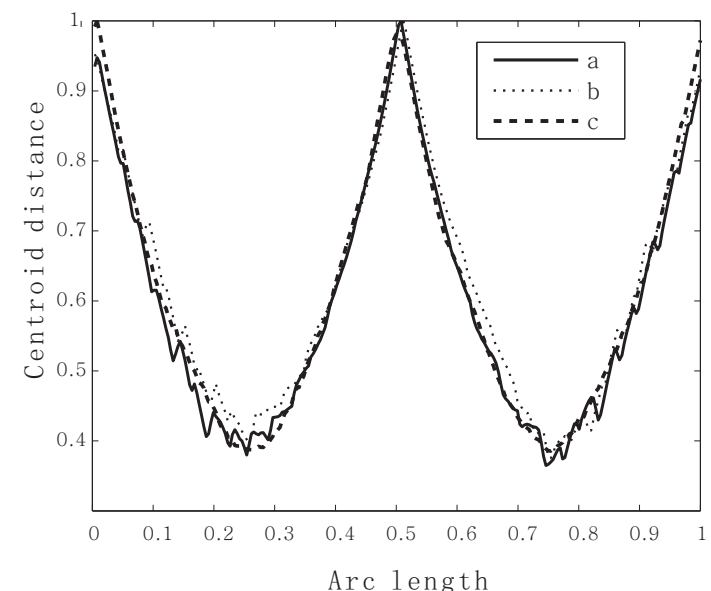

Fig. 2. The centroid distance signatures of three leaf images, where a and b belong to the same class and c belong to another class.

termed multi-scale convexity concavity (MCC) representation, which used the relative displacement of a contour point, with respect to its position in the preceding scale level, to measure the convexity and concavity properties at different scales. This idea is inspired by the observation that smoothing a closed contour will make the convex and concave points move inside and outside the contour, respectively. Alajlan [11] proposed another multi-scale shape descriptor, termed triangle area representation (TAR). This method utilizes the areas of the triangles formed by the boundary points to measure the convexity/concavity of each contour point at different scales, where scale denotes the length of the arc which associates with the triangle formed by the contour point and its two neighbor points. All the above mentioned methods provide powerful discrimination ability for shape based image retrieval because they can not only capture the global shape features, but also reflect the detail information of the shape contour.

However all of the above mentioned methods suffer from an common problem, that is they have very low retrieval efficiency. This problem includes two aspects: (1) Finding one-to-one dense optimal point correspondence between the two shape contours has an expensive computational cost, and some algorithms of high time complexity such as Hungarian algorithm [8] and dynamic programming algorithm [9-11] whose time complexities range from $O\left(N^{2}\right)$ to $O\left(N^{3} \log N\right)$, where $\mathrm{N}$ is the number of the contour points. Therefore, the computational cost of these methods at shape matching stage is very expensive. For example, we conduct an experiment using IDSC [9] to retrieve a leaf image from the Swedish leaf database [12] which contains 1125 images, the experimental results report that it takes about 40 seconds to obtain the retrieval results; (2) Since these methods have to prepare a set of features for each contour points, the number of the features is very large and a feature database of large size is required to store them (Note that for improving retrieval speed, retrieval tasks are usually 
performed on the feature database rather than directly on the image database). For example, IDSC [9] uses about several thousands features for shape matching. Therefore, these methods are not suitable for performing shape retrieval tasks in the large image databases and is also not suitable for those real time applications which require high retrieval efficiency, e.g. online leaf image retrieval. To further improve the retrieval efficient, some recent works [15-17] make an effort to learning similarity-preserving binary codes for efficient similarity search in large-scale image collections. However the effectiveness of resulting binary codes depend on the extracted shape features, i.e. the original shape descriptors. In this work, we focus on shape features extraction and matching.

In this paper, we propose a novel shape descriptor, called multi-level curve segment measures (MLCSM) for fast and effective leaf image retrieval. MLCSM measures the curve segments of different length of the shape contour, we call this scheme muti-level curve segments measures. In the first level, three types of feature, curve bending, convexity, concavity, of all the curve segments of the shape contour whose length is equal to $L / 2$ are measured and the mean value and standard deviation, for each type of feature are recorded, where $L$ is the perimeter of the shape contour. In the jth level curve segment measures, all the curve segments whose length is equal to $L / 2^{j}$ are suffered from the same measure as the first level. The statistical features derived from all the $K$ level measures are combined to form a feature vector to describe the shape, where $K$ is the parameter and refers to the pre-specified number of measure level. This scheme can finely capture the global features and the details of the shape contour. Therefore it can provide a powerful discrimination ability.

The contributions of our work are: (1) we propose a very effective shape description and matching method which present better retrieval accuracy on the leaf image database than the classical Fourier descriptor and the recent methods [9-11]; (2) our method is very compact (only 45 feature is used) and provides a very fast retrieval on leaf image databases. Using our method, it only takes 0.083 seconds to retrieval a leaf image from the Swedish leaf database [12] which contains 1125 images. While the comparable methods [9-11] on the retrieval accuracy require several thousands of features and their time cost range from 18 to 40 seconds. Our method is tested on the Swedish leaf database [12] and the leaf database collected by us from the word which contains 1200 images and 100 plant leaf species. All the experiments show the superiority of our method over the classical Fourier descriptors and other recent methods which reported good retrieval accuracy in the literatures.

The remainder of this paper is organized as follows: Section 2 describe the proposed shape description method. Section 3 gives an analysis of the computational complexity of the proposed method. In section 4 , we present the experimental results and the performance comparisons. Section 5 gives our conclusion. 


\section{Proposed shape description}

\section{$2.1 \quad$ Multi-level curve segment measures}

A shape contour can be represented by a single-closed digital curve $\mathrm{P}$ which is uniformly sampled as a sequence of points $P=\left\{p_{i}\right\}(i=1, \ldots, N)$, where the index $i$ is according to the order of the sample points along the contour in counter-clockwise direction, and $N$ is the number of sample points. Let arc $\widehat{p_{i} p_{j}}$ be a piece of curve segments of curve $\mathrm{P}$ which is started from $p_{i}$ and ended in $p_{j}$ along the counter-clockwise direction. The curve bending, convexity and concavity measures for the arc $\widehat{p_{i} p_{j}}$ are defined as follows:

Definition 1. Let $l$ denote the length of arc $\widehat{p_{i} p_{j}}$ and $b$ be the length of chord $\overline{p_{i} p_{j}}$, the curve bending measure $f_{1}$ for arc $\overline{p_{i} p_{j}}$ is defined as

$$
f_{1}=\frac{b}{l}
$$

Definition 2. Let $S_{L}$ denote the set of all the points of arc $\widehat{p_{i} p_{j}}$ which fall on the left side of the chord $\overline{p_{i} p_{j}}$, and $S_{R}$ denote the set of all the points which fall on the right side of the chord $\overline{p_{i} p_{j}}$, where the 'left' and 'right' are determined by walking from $p_{i}$ to $p_{j}$ along the chord $\overline{p_{i} p_{j}}$. For each point $p_{t}$ of the arc $\widehat{p_{i} p_{j}}$, let $h\left(p_{t}, \overline{p_{i} p_{j}}\right)$ denotes the perpendicular distance from point $p_{t}$ to the chord $\overline{p_{i} p_{j}}$. Then the convexity and concavity measure, $f_{2}$ and $f_{3}$, for arc $\widehat{p_{i} p_{j}}$ are defined as

$$
f_{2}=\sum_{p_{t} \in S_{R}} h\left(p_{t}, \overline{p_{i} p_{j}}\right)
$$

and

$$
f_{3}=\sum_{p_{t} \in S_{L}} h\left(p_{t}, \overline{p_{i} p_{j}}\right)
$$

respectively.

Fig. 3 gives an example to illustrate the convexity / concavity measures for a pieces of curve segment. In Fig. 3, (a) shows a piece of curve segments of shape contour (labeled by heavy line), (c) shows a subdivision of this curve segment, (b) and (d) are their convexity / concavity measures, respectively. From (b), we can see that the measured curve is completely convex $\left(f_{3}=0\right.$ and $\left.f_{2}>0\right)$ relative to its corresponding chord. However the curve segment in (b) have a part of arcs is obviously concave, which can not be described in this measure. The problem can be solved by further measuring the subdivision of the curve segment (See (d) graph ) and more details information of the curve segments will be captured from the further measure. Based on this idea, we propose a multi-level curve segments measures (MLCSM) based shape description. The algorithm of generating MLCSM description is as follows: 


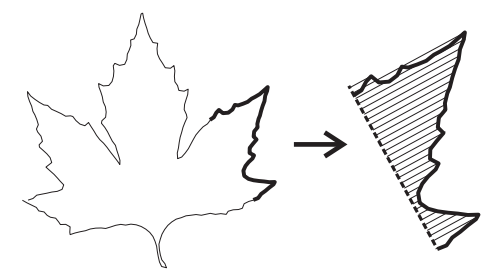

a

b

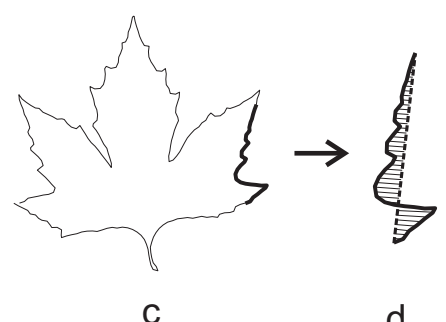

Fig. 3. An example of illustrating the convexity / concavity measures for two pieces of curve segments of the shape contour which have different arc length, where the curve segment in C (labeled by heavy line) is a subdivision of the curve segment in A (labeled by heavy line).

Step 1 Calculate the perimeter of the shape contour $L$. let $K$ be a pre-specified positive number which denote the number of curve segment levels and let $\mathrm{t}=1$ denote the index of level.

Step 2 For each contour point $p_{i}$, start from it to segment a piece of arc whose length is equal to $L / 2^{t}$ from the contour in counter-clockwise direction. Measure the curve bending value $f_{1}(i)$, convexity value $f_{2}(i)$ and concavity value $f_{3}(i)$, of the arc using Eq. 1, Eq. 2 and Eq. 3, respectively.

Step 3 Normalize $f_{1}, f_{2}$, and $f_{3}$ by their respective maximum value.

Step 4 Calculate the mean value and stand deviation of $f_{1}, f_{2}$, and $f_{3}$, respectively and store them as $M_{1, t}, S t_{1, t}, M_{2, t}, S t_{2, t}, M_{3, t}, S t_{3, t}$.

Step 5 let $\mathrm{t}=\mathrm{t}+1$, if $t<=K$ then go to step 2 .

Step 6 Output feature vector $\left\{M_{1, t}, S t_{1, t}, M_{2, t}, S t_{2, t}, M_{3, t}, S t_{3, t}\right\}(t=1, \ldots, K)$.

\subsection{Dissimilarity Measure}

The dissimilarity measure between two shape contours is calculated based on a metric distance between the associate feature vectors. Assume that the feature vectors for shape $\mathrm{A}$ and shape $\mathrm{B}$ are $\left\{M_{1, t}^{A}, S t_{1, t}^{A}, M_{2, t}^{A}, S t_{2, t}^{A}, M_{3, t}^{A}, S t_{3, t}^{A}\right\}(t=$ $1, \ldots, K)$ and $\left\{M_{1, t}^{B}, S t_{1, t}^{B}, M_{2, t}^{B}, S t_{2, t}^{B}, M_{3, t}^{B}, S t_{3, t}^{B}\right\}(t=1, \ldots, K)$, respectively. Let $d_{j}=\sum_{t=1}^{K}\left(\left|M_{j, t}^{A}-M_{j, t}^{B}\right|+\left|S t_{j, t}^{A}-S t_{j, t}^{B}\right|\right)$. The dissimilarity measure between shape $\mathrm{A}$ and shape $\mathrm{B}$ is defined as

$$
D(A, B)=d_{1}+w_{1} * d_{2}+w_{2} * d_{3},
$$

where $w_{1}$ and $w_{2}$ are weight parameters.

Many global features such as circularity, eccentricity, aspect and so on can reflect the valuable information about the global properties which can benefit the shape retrieval, therefore many methods $[11,13]$ incorporated some of them to improve the performance. To further increase the discrimination ability for the leaf shape, we also incorporate three global features, solidity $(\mathrm{S})$, eccentricity (E) and rectangularity $(\mathrm{R})$ to our method. Let $D_{g}(A, B)=(|S(A)-S(B)|+$ 
$|E(A)-E(B)|+|R(A)-R(B)|)$. The final dissimilarity measure incorporated by global features is defined as follows:

$$
D_{f}(A, B)=D(A, B)+w_{3} * D_{g}(A, B),
$$

Where $w_{3}$ is weight parameter.

\subsection{The desirable properties of the proposed MLCSM}

From the above algorithm of generating MLCSM, at the first level (i.e. $t=1$ ), MLCSM measures the long curve segments which make the captured feature are not insensitive to the details of the shape contour, therefore, at this level, the measures tend to capture the global features of shape contour. When the index of measure level become large, the length of the measured curve segments will become small which make the measurement have the higher ability of capturing the small details. So, MLCSM can finely capture the global and local features and provide a powerful discrimination ability. We still use the three leaf images shown in Fig. 2 as example. Since centroid distance signature is not sensitive to the the details of the shape contour, so FDs derived from centroid distance give a false matching. While MLCSM can finely capture the details of the shape contour, so the sawtooth feature of the shape boundary of leaf (a) and leaf (b) can be reflected by MLCSM. Here, we present the matching result by our method using Eq. 4 as follows: $\mathrm{D}(\mathrm{a}, \mathrm{b})=0.9109, \mathrm{D}(\mathrm{a}, \mathrm{c})=1.5965, \mathrm{D}(\mathrm{b}, \mathrm{c})=1.7661$. From the matching result, we can see that MLCSM presents a correct matching.

A good shape description should be invariant to translation, scaling and rotation. This is because these geometry transforms do not change the shape of an object. Since the curve bending, convexity and concavity measures of a piece curve segment have nothing to do with the position of shape contour. Therefore MLCSM is invariant to translation. At the step 3 of the above algorithm, we normalize $f_{1}, f_{2}$, and $f_{3}$ using their respective maximum value. So, MLCSM is also invariant to scaling. As for rotation, when a piece of curve segment is rotated, according to Eq. 1-3, the value of $f_{1}, f_{2}, f_{3}$ is obviously not modified. When the starting point of the shape contour is changed by rotating the shape contour, MLCSM is also invariant. This is because MLCSM calculates the mean value and standard deviation of $f_{1}, f_{2}, f_{3}$ which make them invariant to starting point change. Therefore MLCSM is invariant to rotation transform.

A good shape description should also be as compact as possible and robust to noise. At each level of curve segment measure, we extract 6 features, so there are total of $6 K$ features are extracted, where $K$ is the number of the levels and is far less than to the number of contour points. In our experiments, we set $\mathrm{k}=7$ and only use 42 features to achieve higher retrieval accuracy. While many recent methods $[10,8,9]$ require several thousands of features to support their high retrieval accuracies. Since we use the mean value and standard deviation of $f_{1}, f_{2}, f_{3}$ to describe the shape, they are global calculations, therefore MLCSM is not insensitive to noise. 


\section{Computational complexity}

For image retrieval, especially for retrieval on the large image database or online retrieval, low computational complexity is an important characteristic for a desirable shape descriptor. In this section, we analysis the computational complexity of the proposed method. For a piece of curve segments of $M$ points, i.e. the length of the segments, the complexity of measuring the curve bending, convexity and concavity is $O(M)$. Assume that the shape contour have $N$ sample points and the number of pre-specified measure level is $K$. On the jth level of curve segment measure, all the arcs whose length $M$ is equal to $N / 2^{j}$ are measured, the complexity of performing these measures is $O\left(N^{2} / 2^{j}\right)$. Therefore the total complexity of features extraction is $O\left(N^{2}\left(1 / 2+\ldots,+1 / 2^{k}\right)\right)=O\left(N^{2}\left(1-1 / 2^{k}\right)\right)=O\left(N^{2}\right)$. While many recent methods have higher computational complexity of features extraction than our method. For example, MCC [10] is $O\left(K N^{2}\right)$, Shape context method (SC) [8] and Inner distance method $[9]$ are $O\left(N^{3}\right)$, where $K$ is the number of scale levels and $N$ is the number of contour points.

Since on shape retrieval, the shape feature are extracted only once and can be pre-extracted to be stored in the feature databases. While shape matching is conducted many times. For each query shape, all the shapes in the database will be matched with this shape. Therefore the retrieval efficiency mainly depend on the efficiency of shape matching. At the shape matching stage, our method use a metric distance to measure the shape similarity, so the computational complexity depend on the length of the feature vector. Since at each curve segment measure level, we extract 6 features, the total length of feature vector of our method is $6 \mathrm{~K}$. Therefore the computational complexity of our method at shape matching stage is $O(6 K)=O(K)$, where $k$ is the number of curve segment measures and is far less than the number of the sample contour points $N$. As for many recent methods with higher retrieval accuracy, such as Shape context [8], TAR[11], MCC[10], Inner distance [9], and Visual Parts [14], their Computational complexity of shape matching range from $N^{2}$ to $N^{3} \log N$.

\section{Experimental results}

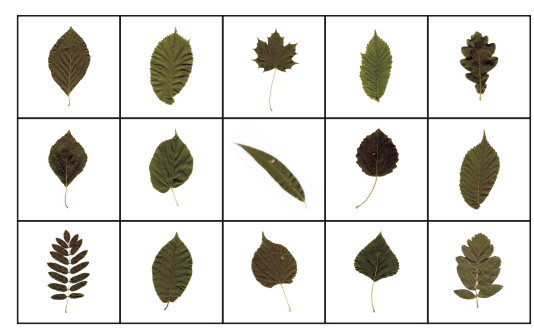

Fig. 4. Fifteen samples from the Swedish leaf databases, one image per species. 


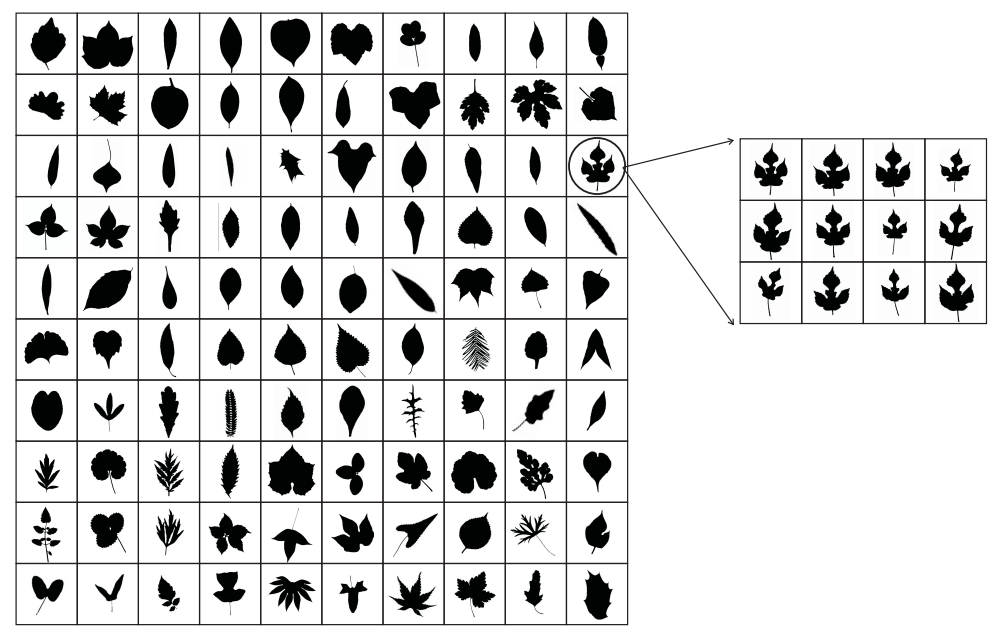

Fig. 5. Leaf image database built by ourselves which contains 100 species with 12 instances each.

In this section, the performance of the proposed multi-level curve segment measures (MLCSM) shape description is demonstrated on two leaf image databases. The first database is the well-known Swedish leaf database which contains isolated leaves from 15 different Swedish tree species (see Fig. 4)with 75 leaves per species for a total of 1125 images. The second one is a leaf shape database which consists of 1200 leaves of 100 plant species with 12 samples each (see Fig. 5). All of the samples in this database are collected by ourselves from the wild. From the two leaf databases, we can see that both of them contain some species which have many very similar shapes.

In the experiments, we compared the following algorithms using the above two leaf databases: (1) The classical FDs derived from centroid distance (CD) which has been proved to be suitable for shape retrieval by Zhang et al [6], (2) Multi-scale convexity concavity (MCC) representation [10], (3) Triangle-area representation (TAR) [11], (4) Inner distance + shape contexts (IDSC) [9], (5) The proposed methods: MLCSM and MLCM+three global features. The experimental platform is a PC with Intel Core-2 Duo 2.8G.Hz CPU running Windows XP. The FDs and our method are coded in Matlab 6.5. Because of the high calculational complexity of shape matching for MCC, TAR and IDSC, we code them in the combination of Matlab and $\mathrm{C}$ program language, in which the procedure of shape matching are coded in $\mathrm{C}$ program language.

The retrieval accuracy is measured by the so-called Bullseye test $[9,11,10]$. This evaluated method is: each shape in the database is taken as a query and matched to all the shapes in the database. The top $2 \times M$ matches are counted, where $M$ is the number of shapes in each category. At most, $\mathrm{M}$ of the $2 \times M$ candidates are correct matches. The retrieval accuracy is the ratio of the number of correct matches of all images to the total maximum number of correct matches 
Table 1. Retrieval results of different methods on the Swedish leaf database.

\begin{tabular}{llcl}
\hline Method & The number of features & $\begin{array}{c}\text { Retrieval accuracy } \\
(\%)\end{array}$ & $\begin{array}{c}\text { Retrieval speed } \\
(\mathrm{MS})\end{array}$ \\
\hline FDs & 20 & 69.13 & 3 \\
TAR [11] & 8067 & 84.23 & 28234 \\
MCC [10] & 1280 & 84.17 & 18579 \\
IDSC [9] & 9600 & 88.51 & 37141 \\
MLCSM & 42 & 86.47 & 83 \\
MLCSM+Global & 45 & 88.65 & 83 \\
\hline
\end{tabular}

(which is $M \times M \times N$ ), where $\mathrm{N}$ is the number of the classes of the shapes. The retrieval speed is the average time cost of retrieval of each query (include the time of feature extraction of query shape). The parameters of our method is as follows: the number of uniformly sampled points of the shape contour is 256 , the number of the levels of curve segment measures is set to 7 . The parameters of Eq. 4 and Eq. 5, i.e. weight values, are $w_{1}=1.2, w_{2}=1.0$ and $w_{3}=1.1$.

Table 2. Retrieval results of different methods on the leaf database built by ourselves.

\begin{tabular}{llcl}
\hline Method & The number of features & $\begin{array}{c}\text { Retrieval accuracy } \\
(\%)\end{array}$ & $\begin{array}{c}\text { Retrieval speed } \\
(\text { MS })\end{array}$ \\
\hline FDs & 20 & 60.12 & 4 \\
TAR [11] & 8067 & 77.66 & 36484 \\
MCC [10] & 1280 & 77.10 & 20841 \\
IDSC [9] & 9600 & 85.64 & 39969 \\
MLCSM & 42 & 84.62 & 84 \\
MLCSM+Global & 45 & 86.12 & 84 \\
\hline
\end{tabular}

The retrieval results of different methods on the two leaf databases are shown in Table 1 and Table 2, respectively. From Table 1 and Table 2, we can see that among all the competing methods, Fourier descriptors (FDs) has the least number of features and provide the fastest retrieval, unfortunately, it also has the lowest retrieval accuracy. TAR, MCC and IDSC has good retrieval accuracy, however their retrieval speed is very slow because of the very expensive calculational cost and the number of used features is very large which make them not suitable for leaf image retrieval especially online leaf image retrieval. While our methods achieve about 20 percents higher than FDs on retrieval accuracy and the number of used features and retrieval speed is obviously acceptable. The experimental results also show that our method achieves higher retrieval 
accuracy than the recent methods TAR and MCC even without using the three global features. While the global parameters are incorporated in the matching function, our method outperforms all the competing methods in terms of the retrieval accuracy.

\section{Conclusion}

In this paper, we have presented a novel shape representation and matching methods based on multi-level curve segments measures (MLCSM). MLCSM extracts the statistical features of shape contour via measuring the curve bending, convexity and concavity of curve segments with different levels to describe the shape. This method is very compact and can finely capture the global and local features of the shape contour. The metric distance between the acquired feature vectors is used to measure the shape dissimilarity. The three global features, solidity, eccentricity and rectangularity, are incorporated in the shape dissimilarity measure to further increase the discrimination ability. The retrieval experimental results on the two leaf image databases show that as compared with several state-of-the-art methods, MLCSM has higher degree of retrieval accuracy and retrieval efficiency. In our future work, we will consider learning similarity-preserving binary codes based on the proposed shape descriptor to further improve the efficient of plant leaf image retrieval.

\section{References}

1. Du, J.X., Wang, X.F., Zhang, G.J. Leaf shape based plant species recognition. Allied Mathematics and Computation. 185(2007) 883-893

2. Zhang, D.S., Lu, G.J. Review of shape representation and description techniques. Pattern Regcognition. 37(2004)1-19.

3. Zahn,T., Roskies, R.Z. Fourier descriptors for plane closed curves. IEEE Trans. Comput. 21(1972)269-281.

4. Kauppinen, H., Seppanen, T., Pietikainen, M. An experimental comparison of autoregressive and Fourier-based descriptors in 2-D shape classification. IEEE Trans.PAMI. 17(1995): 201-207.

5. Mehtre, B.M., Kankanhalli, M.S., Lee W.F. Shape measures for content-based image retrieval: a comparison. Information Processing \& Management. 33(1997) 319-337.

6. Zhang, D.S., Lu, G.J. A comparative study of curvature scale space and Fourier descriptors. Journal of Visual Communication and Image Representation. 14(2003) 41-60.

7. Zhang, D.S., Lu, G.J. Study and evaluation of different Fourier methods for image retrieval. Image and Vision Comput. 23(2005)33-49.

8. Belongie, S., Malik, J., Puzicha, J. Shape matching and object recognition using shape contexts. IEEE Pattern Anal. Mach. Intell. 24(2002)509-522.

9. Lin, L., Jacobs, D. Shape classification using the inner-distance. IEEE Trans. Pattern Anal. Machine Intell. 29(2007)286-299. 
10. Adamek, T., O'Connor, N.E. A multiscale representation method for nonrigid shape with a single closed contour. IEEE Trans. on Circuits and systems for video technology. 14(2004) 742-743.

11. Alajlan, N., Rube, I.E., Kamel, M.S, Freeman, G. Shae retrieval using trianglearea representation and dynamic space warping. Pattern Recognition. 40(2007) 1911-1920.

12. Soderkvist, O. Computer vision classification of leaves from swedish trees. Master thesis, Linoping Univ., 2001.

13. Abbasi, S., Mokhtarian, F., Kittler,J. Curvature scale space image in shape similarity retireval. Multimedia Syst. 7(1999) 467-476.

14. Latecki, L.J., Lakamper, R. Shape similarity measure based on correspondence of visual parts. IEEE Trans. Pattern Anal. Mach. Intell. 22(2000)1185-1190.

15. Gong, Y.C., Lazebnik, S., Gordo, A., Perronnin, F. Iterative Quantization: a procrustean approach to learning binary codes for large-scale image retrieval. International Conference on Computer Vision and Pattern Recognition, pp. 817-824, 2011.

16. Weiss, Y., Torralba, A., Fergus, R. Spectral Hashing. In Proceedings of the 22nd Annual Conference on Neural Information Processing Systems (NIPS), pp. 17531760, 2008.

17. Wang, J., Kumar, S., S.F., Chang. Semi-supervised hashing for scalable image retrieval. International Conference on Computer Vision and Pattern Recognition, pp. 3424-3431, 2010. 